\title{
Design and laboratory performance of a fiber-fed Fourier transform spectrograph based on off-the-shelf components for astronomical medium and high-resolution spectroscopy
}

\author{
Pornapa Artsang $\odot, \stackrel{\text { a,b }}{ }$ Christophe Buisset, ${ }^{\mathrm{b}, *}$ Panomsak Meemon $\odot, \stackrel{\mathrm{a}, \mathrm{c}, *}{ }$ \\ Pakakaew Rittipruk $\odot,{ }^{\text {b }}$ Sirinrat Sithajan, ${ }^{\text {b }}$ Boonracksar Soonthornthum, ${ }^{\text {b }}$ \\ and Saran Poshyachinda ${ }^{b}$

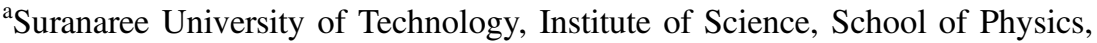 \\ Muang Nakhon Ratchasima District, Nakhon Ratchasima, Thailand \\ ${ }^{b}$ National Astronomical Research Institute of Thailand, Center for Optics and Photonics, \\ Donkaew Sub-District, Maerim District, Chiang Mai, Thailand \\ ${ }^{\mathrm{c} S}$ Suranaree University of Technology, Center of Excellent in Advanced Functional Material, \\ Muang Nakhon Ratchasima District, Nakhon Ratchasima, Thailand
}

\begin{abstract}
We present the design and performance verification of a fiber-fed Fourier transform spectrograph (FTS) for spectroscopy in the optical band with the ability to reach a maximum optical path difference of $15 \mathrm{~mm}$ and allowing for an adjustable spectral resolution $(\lambda / \Delta \lambda)$ between 1 and 15,000. The designed FTS system was successfully constructed using only off-the-shelf optical components. The technique for correction of the phase distortion in the FTS using a metrology interferogram and cubic spline interpolation was investigated and discussed. The contrast performance and the instrument line shape of the FTS were measured and analyzed. To further verify the performance of the developed system, the absorption spectrum of the sunlight was measured and compared with a synthetic model with identified telluric and absorption lines. The result shows that the developed FTS can detect the absorption lines with a spectral resolution close to 15,000. ( ) The Authors. Published by SPIE under a Creative Commons Attribution 4.0 International License. Distribution or reproduction of this work in whole or in part requires full attribution of the original publication, including its DOI. [DOI: 10.1117/1.OE.61.1.014104]
\end{abstract}

Keywords: Fourier transform spectrograph; spectroscopy; optical path difference; cubic spline interpolation.

Paper 20211060 received Sep. 20, 2021; accepted for publication Dec. 22, 2021; published online Jan. 11, 2022.

\section{Introduction}

The spectroscopy technique is used by astronomer to study the universe to find out the physical properties or determine the chemical composition and radial velocities of stellar objects. ${ }^{1} \mathrm{~A}$ Fourier transform spectrograph (FTS) is one of the most challenging instruments to be applied in astronomy. The history of FTS in ground-based astronomy has been nicely summarized by Maillard et al. ${ }^{2}$ and Drissen et al. ${ }^{3}$ The use of FTS started in the 1970s and in 1980s when the FTS was installed on the Kitt Peak's Mayall 4-m telescope. Since then, FTSs have been widely used to provide spectra in the near-infrared of both single-point and extended objects. ${ }^{4,5}$

Recently, the highly effective imaging FTS that operates in the optical domain was developed and installed on large telescopes. First, the Spectromètre Imageur de l'Observatoire du Mont-Mégantic was installed on the focal plane of the 1.6-m telescope of the Mont Mégantic Observatory. More recently, the SITELLE was installed on the 3.6-m Canada-France-Hawaii Telescope. This instrument was commissioned in 2015 and has provided data cubes over the spectral domain $\left[350 \mathrm{~nm}, 900 \mathrm{~nm}\right.$ ] with a spectral resolution adjustable ${ }^{6}$ between 1 and 10,000

*Address all correspondence to Christophe Buisset, christophe@narit.or.th; Panomsak Meemon, panomsak@sut.ac.th 
over a field-of-view of $11^{\prime} \times 11^{\prime}$ with a seeing-limited angular resolution close to $0.8^{\prime \prime}$. These FTSs are installed directly at the focal plane of the telescopes. The design thus involves only large and custom-made optics together with complex mechanical structures and active mechanisms to guarantee the performance in operational conditions. These constraints lead to massive custom-made instruments that are difficult to manufacture and very expensive.

Most of the current fiber-fed medium and high-resolution spectrographs mounted on large telescopes are Echelle spectrographs. ${ }^{7,8}$ One of the most famous examples is the HARPS spectrograph mounted on the 3.6-m telescope of La Silla observatory at the European Southern Observatory. This instrument has provided high quality spectra and has made the discovery of several new exoplanets and other astronomical phenomena possible. ${ }^{9,10}$ However, these spectrographs usually suffer from several limitations, such as instrument volume, complexity, cost, throughput, and sensitivity to fiber modal noise.

In the fiber-fed Echelle spectrograph, the fiber acts as an entrance slit from which the beam is focused onto the dispersive elements. The focal length is adjusted to reach the desired spectral resolution. In an extended fiber core, a larger spectrograph volume is thus required due to the increasing of focal length. The diameter of the fiber of an Echelle spectrograph is adjusted to match the size of the star image provided by the telescope and defined by the seeing conditions. The collimator focal length is then adjusted to reach the specified spectral resolution and the fiber core diameter. Therefore, the focal length defines the size of the optical surfaces and thus the instrument volume and cost. For example, in HARPS, the grating length is $125 \mathrm{~mm}$, and the full instrument requires a vacuum vessel with a volume close to $2 \mathrm{~m}^{3}$ to stabilize the instrument environment to reach the specified performance. The cost is thus usually very high, and only large observatories with massive budget resources can afford the development of such instruments.

In addition, the throughput of Echelle spectrographs is limited by the size of the input fiber core diameter (matched to fit the seeing conditions), the grating efficiency, and the optics transmission. There is a trade-off between fiber core diameter and system efficiency. For example, the HARPS spectrograph has two operating modes, i.e. the standard (HAM) mode and the high efficiency (EGGs) mode. The HAM mode uses fibers with a core diameter of $70 \mu \mathrm{m}$, covering the sky aperture of $1^{\prime \prime}$, and providing the accuracy of radial velocity of $1 \mathrm{~m} / \mathrm{s}$. In the EGGs mode, the fibers with a core diameter of $100 \mu \mathrm{m}$ are used, covering a sky aperture of 1.4", with a throughput gain factor of 1.75 as compared with that of the HAM mode. However, EGGs mode provides the accuracy of radial velocity of $3 \mathrm{~m} / \mathrm{s}$, which is lower than that of the HAM mode. The maximum transmission of HARPS including the limited core diameter and the spectrometer transmission is close to $12 \% .^{10}$

Moreover, the Echelle spectrograph provides an image of the fiber output face at each wavelength. The irradiance measured by the detector is thus defined by the modes propagation through the fiber. The vibrations, constraints, and temperature variations of the operational conditions change the modes propagation, thus inducing a temporal modification of the spectrograph point spread function that impacts the instrument accuracy. ${ }^{11}$ For this reasons, stateof-the-art high-resolution spectrographs usually use some mode scramblers to mitigate the effect of mode propagation on the instrument stability.

In this paper, we present the design and performance of a fiber-fed FTS for low-, medium-, and high-resolution spectroscopy to be equipped on the $2.4 \mathrm{~m}$ Thai National Telescope (TNT). ${ }^{12}$ Based on the availability and cost of off-the-shelf optical components, the instrument was designed to cover a spectral measurement in the range of $400 \mathrm{~nm}$ to $1 \mu \mathrm{m}$. A targeted spectral resolution at wavelength $\lambda$ as defined by ${ }^{1} R=\lambda / \Delta \lambda$, where $\Delta \lambda$ represents the smallest wavelength resolved, will be adjustable between 1 and 70,000. A targeted signal-to-noise ratio (SNR) will be higher than 10 on stars of magnitude $m \approx 6$. The potential advantages are as follows: a compact and cost-effective system using only commercial components, a large diameter of the scientific fiber core, a high throughput limit, and insensitivity to fiber modal noise. The design and implementation of the instrument are described. In addition, a technique for signal processing of the developed FTS system based on the use of a metrology interferogram and spline interpolation is presented and discussed. Moreover, the contrast performance and spectral resolution of the instrument are analyzed and presented. The instrument line shape (ILS) is verified using several clear absorption peaks in the I-band of the measured Sun's spectrum. 


\section{Instrument Concept and Prototype Design}

The TNT is a 2.3-m Ritchey-Chretien Telescope mounted on an Alta-Azimuthal mount. This instrument is installed at the Thai National Observatory located at the altitude $2457 \mathrm{~m}$, latitude 18.57 N, and longitude 98.48 E, as shown in Figs. 1(a) and 1(b). In this observatory, the seeing in median conditions is better than $\delta \theta_{\text {seeing }} \approx 2^{\prime \prime}$. The TNT collects the starlight beam with autoguiding and directs this beam toward a guiding and injection unit (GIU) and the scientific optical fiber, as shown in Fig. 1(c). In the fiber-fed spectrograph system, the size of the fiber core that is coupled to the telescope is adjusted to fit the size of the star image at the telescope focus. The numerical aperture $(N A)$ of the GUI is adjusted to be equal to $N A$ of the fiber as ${ }^{13} N A=0.22$, and the TNT clear aperture diameter $(D)$ is ${ }^{14} 2.3 \mathrm{~m}$. The effective focal length of the TNT combined with GIU is calculated as $f_{\text {eff }} \cong D /(2 \cdot N A)=5.2 \mathrm{~m}$. The image of the star can be estimated from the seeing condition and the telescope focal length ${ }^{15} f_{\text {eff }} \cdot \delta \theta_{\text {seeing }}=50.4 \mu \mathrm{m}$. Therefore, we selected an optical fiber with a core diameter $\Phi_{c}=50 \mu \mathrm{m}$.

The fiber directs the light beam from the TNT GIU to a Y-coupler that comprises two output fibers. The first fiber directs a fraction of the flux toward a photodetector to measure the real time variations of the flux propagating through the fiber. The second fiber directs the light toward the FTS, as shown in Fig. 2. The fiber output face is located at the focus of the lens L1 that collimates and directs the scientific beam toward the 50:50 beam splitter (BS).

The BS separates the incident beam into two beams. On channel 1, the BS reflects the beam toward dynamic mirror M1. This mirror is mounted on a linear translating stage and is moved by a few centimeters during the data acquisition. On channel 2, the BS transmits the beam toward static mirror M2, which reflects the beam back to the BS.

The BS combines the channel 1 and 2 beams and provides two output beams. One beam is incident on the spectral filter and the balanced photoreceiver. The other beam is incident on the reflecting prism $\mathrm{P} 1$, which directs the beam toward the spectral filter and the second entrance of the balanced photoreceiver. The spectral filter located in front of the balanced photodetector aims at (1) reducing the photon noise by restricting the spectral extent of the beam incident on the detector and (2) suppressing the leak of the metrology signal induced by the scattering, diffraction, and spurious reflections.

The FTS is included on the metrology channel to monitor the displacement of the dynamic mirror during the data acquisition. This corrects for the M1 displacement nonlinearities during the observations. The metrology source is made of a stabilized laser that injects a beam at the wavelength $\lambda_{\text {ref }}=633.178 \mathrm{~nm}$ into a single mode fiber (SMF). The SMF output face is located at the focus of the lens L2. The beam collimated by L2 is directed toward prism P2.

The direction of the beam reflected by $\mathrm{P} 2$ is similar to the direction of the scientific beam incident on the FTS. The optical path of the metrology beam through the interferometer is thus similar to the scientific beam's optical path. One detector located at the FTS metrology channel output records the fringe pattern during the data acquisition. The metrology channel collects data at the sampling frequency of $50 \mathrm{kHz}$ with an integration time of $4.2 \mathrm{~s}$, thus providing $\sim 10$ points
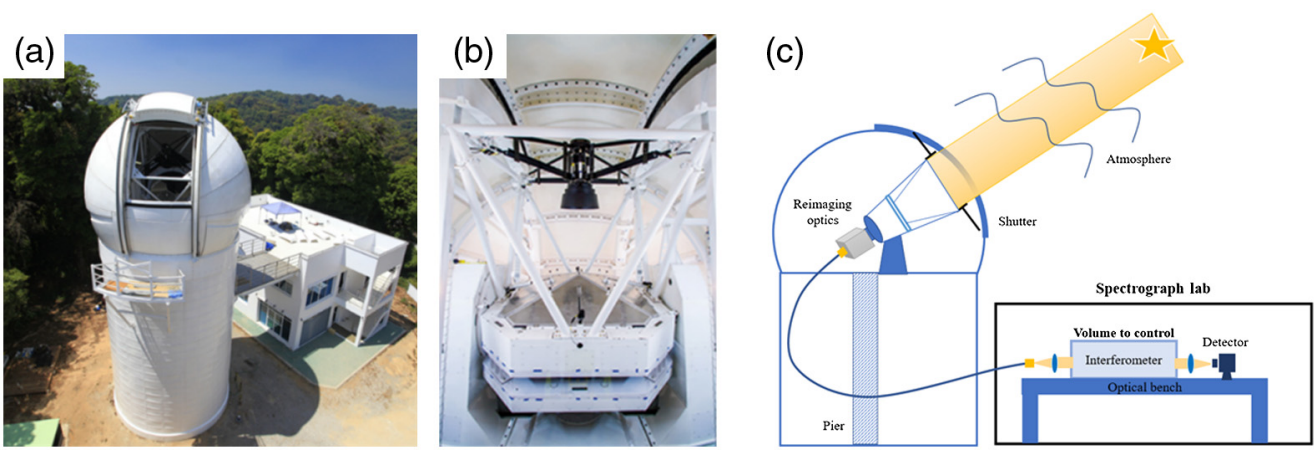

Fig. 1 (a) The Thai National Observatory located on the Doi-Inthanon mountain at the altitude 2,457 m, latitude $18.57 \mathrm{~N}$, and longitude $98.48 \mathrm{E}$. (b) The TNT installed on an Alta-Azimuthal mount inside the dome. (c) Schematic concept of the proposed fiber-fed FTS system. 


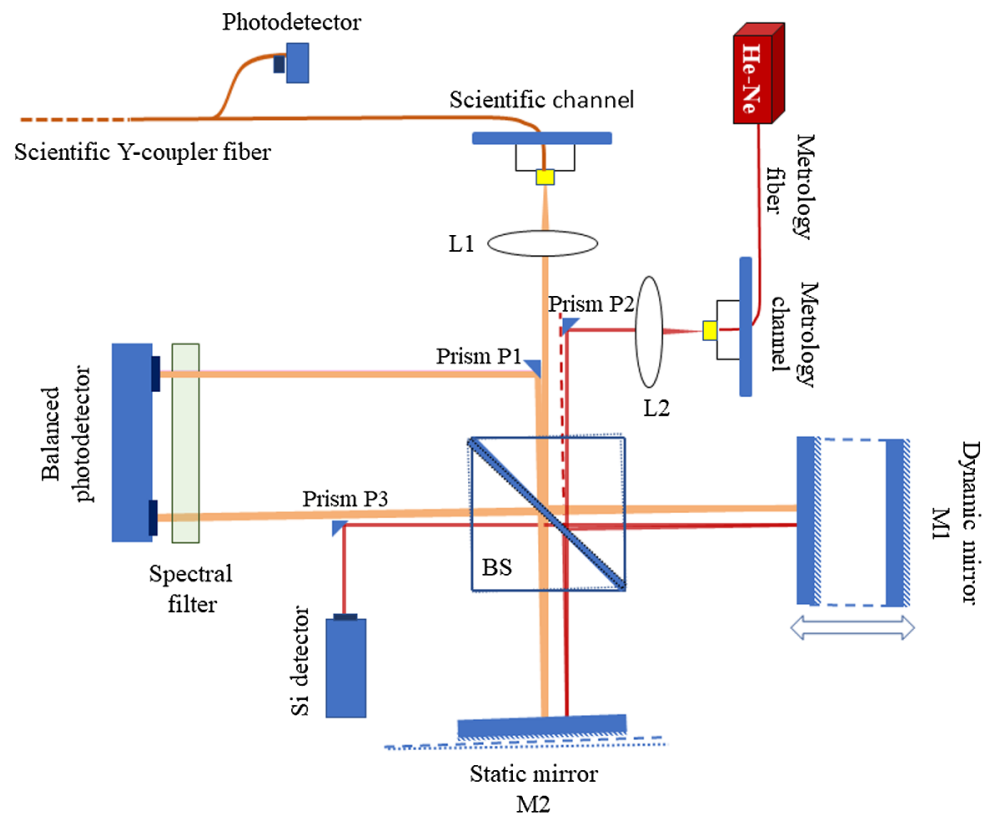

Fig. 2 Schematic of the proposed FTS concept. The scientific beam is represented in orange, and the metrology beam is represented in red.

per fringes. The sampling time interval in both the scientific and metrology paths is $20 \mu \mathrm{s}$, which corresponds to an optical path difference (OPD) error of $36 \mathrm{~nm}$ at a translating stage speed of $1.8 \mathrm{~mm} / \mathrm{s}$. This OPD error is negligible when compared with the wavelengths transmitted by the spectral filter and should not affect the spectrum provided by the FTS.

Figure 3(a) shows the laboratory setup of the spectrometer that has been developed at the NARIT Center for Optics and Photonics using a large core scientific optical fiber and only offthe-shelf optical surfaces and mechanical and electronical components. To verify the performance of the system, we concentrate the sunlight on the entrance face of the scientific fiber using an achromatic doublet, as shown in Fig. 3(b). The scientific fiber directs the light toward the setup comprising the components as shown in Fig. 3(a). The lists of the components for both the scientific and metrology channels are provided in Tables 2 and 3, respectively,

\section{(a)}

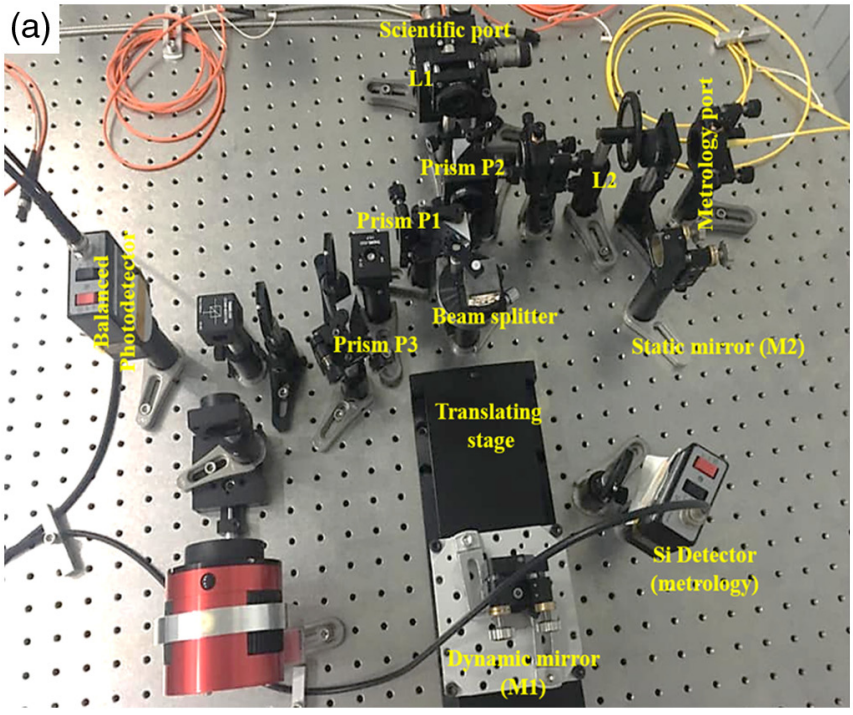

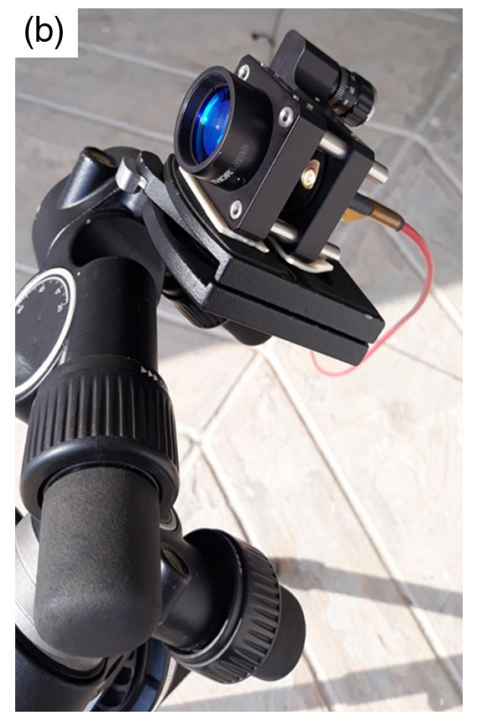

Fig. 3 (a) The FTS prototype assembled at NARIT Center for Optics and Photonics. (b) Setup used to concentrate the sunlight at the scientific fiber entrance. 
in Appendix A. The coupling of the fiber can be adjusted to reduce the flux at the fiber output before entering the system to quantify the minimum detectable flux level of the current implemented system. We quantified that the minimum flux level entering the current setup that allowed for spectrum measurement with SNR $>10$ is $\sim 1 \mu \mathrm{W}$. This input flux level was used for all measurements presented in this paper. As part of our plan, to improve the sensitivity of the system, a phase-lock amplifier is implemented. We already verified that the current detector coupled with the phase lock amplifier [Model SR860, Stanford Research Systems] is able to provide an SNR of 400 for an incident flux of $4 \mathrm{nW}$ for an integration time of $1 \mathrm{~ms}$.

The throughput of the spectrometer is defined by

$$
T_{\text {Spectro }}=T_{\text {Slit }} \times T_{\text {Coupler }} \times T_{\text {Fiber }} \times T_{\text {FTS }},
$$

where $T_{\text {Slit }}$ is the throughput due to the limited size of the fiber core. In our case, the core diameter is adjusted to collect the full star flux by taking account of the seeing and tracking accuracy. We thus assume that $T_{\text {Slit }}=1$. We assume that this injection unit will use (1) optical lenses of coating optimized for the [400 nm, $1 \mu \mathrm{m}]$ and (2) a BS 90:10 to reflect 10\% of the starlight toward a camera for guiding and tracking purposes. $T_{\text {Coupler }}$ is the transmission of the Y-coupler used to monitor the variation of the star flux injected in the fiber and due to the variations of the atmosphere transmission. We assume that $T_{\text {Coupler }}=50 \% . T_{\text {Fiber }}$ is the transmission of the fiber that is taken to be equal to 1 (ideal case) in this study.

$T_{\text {FTS }}$ is the transmission of all optical components between the scientific fiber output face and the detector

$$
T_{\mathrm{FTS}}=T_{\mathrm{L} 1} \times R_{\mathrm{M}} \times\left(R_{\mathrm{BS}}+T_{\mathrm{BS}}\right)^{2} \times T_{\mathrm{SF}},
$$

where $T_{L 1}$ is the transmission of the lens $\mathrm{L} 1$ and $R_{\mathrm{M}}$ is the reflectivity of mirrors M1 and M2, which are assumed to be identical. $R_{\mathrm{BS}}$ and $T_{\mathrm{BS}}$ are the BS's reflection and transmission, respectively. $T_{\mathrm{SF}}$ is the transmission of the spectral filter. We assume in this calculation that the prism that folds the beam has a reflection coefficient of 1 .

Figure 4 shows the theoretical throughput of the FTS equipped with an $I$-band photometric filter obtained from Eq. (2) at the central wavelength of $800 \mathrm{~nm}$ and full width at half maximum (FWHM) of $160 \mathrm{~nm}$ in the black solid line and the transmissions of each optical component separately plotted in the dashed lines. We notice that the theoretical throughput reaches $80 \%$ in the wavelength region of 760 to $840 \mathrm{~nm}$. We measured the throughput of our FTS setup

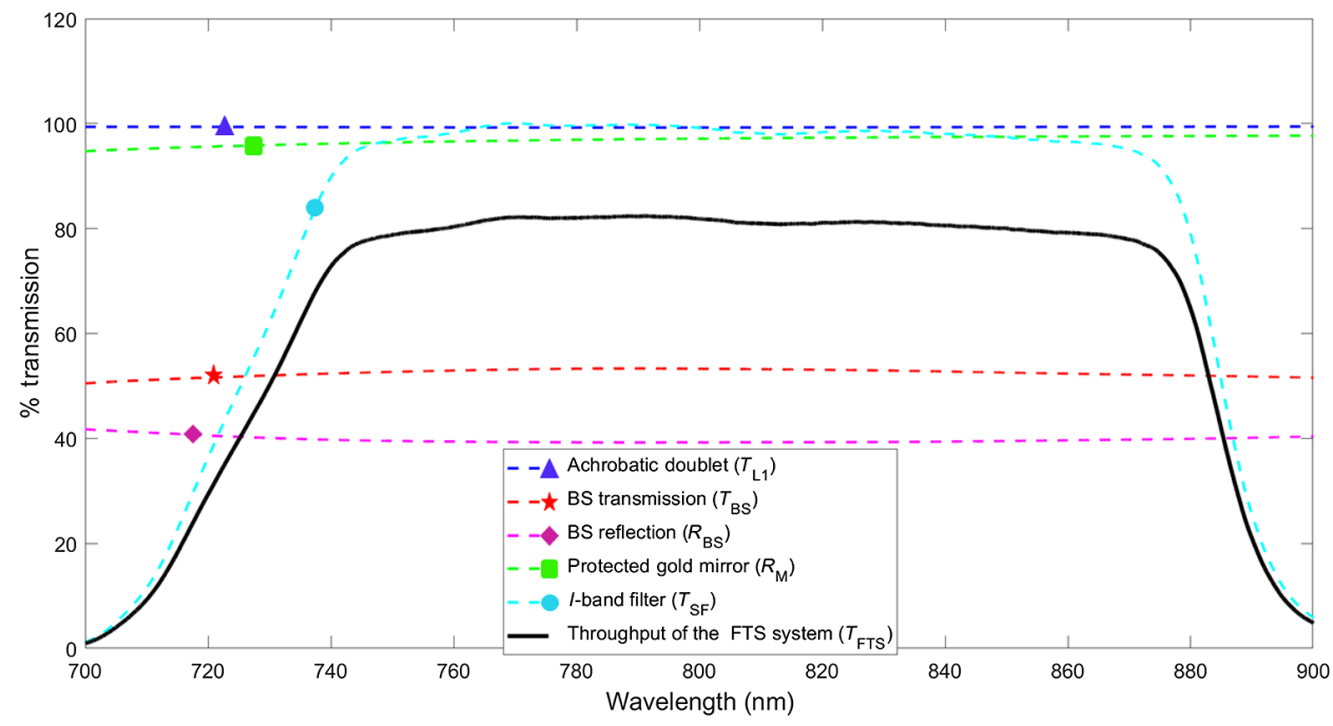

Fig. 4 The theoretical transmission variations of each individual component in the FTS system are separately plotted and in dashed lines (blue with rectangular mark: $T_{\mathrm{L} 1}$, red with star mark: $T_{\mathrm{BS}}$, magenta with diamond mark: $R_{\mathrm{BS}}$, green with rectangular mark: $R_{\mathrm{M}}$, cyan with circular mark: $T_{\mathrm{SF}}$ ). The total transmission throughput of FTS system $\left(T_{\mathrm{FTS}}\right)$ is plotted in the black solid line. 
in the I-band and found that $T_{\mathrm{FTS} \text {,measure }} \approx 85 \%$, which is slightly above but still in line with our predictions. Using Eq. (1), we estimate that the throughput of the full system at the wavelength of $800 \mathrm{~nm}$ is $T_{\text {Spectro }} \approx 42 \%$.

\section{Processing Method}

The processing algorithm used to extract the spectrum from the raw intensity measurements is based on the method presented by Roy et al. ${ }^{16}$ This method comprises three steps, described hereafter. As shown in Fig. 6(a), first, we simultaneously record the scientific and metrology signals shown in Fig. 5. Second, we detect the location of the metrology signal peaks and valleys by upsampled spline interpolation to improve accuracy. ${ }^{17}$ The OPD, which is quantified as twice the mirror displacement, reached at a given metrology signal peak measured at the instant time stamp $T_{\mathrm{PV}, K}$ is called $\mathrm{OPD}_{\mathrm{PV}, K}$, where $K=1, \ldots, N$. The OPD on the preceding signal valley measured at the instant $T_{\mathrm{PV}, K-1}$ is called $\mathrm{OPD}_{\mathrm{PV}, K-1}$, as shown in Fig. 6(b). We thus use the fact of the time-position relation that $\mathrm{OPD}_{\mathrm{PV}, K}-\mathrm{OPD}_{\mathrm{PV}, K-1}=0.5 \lambda_{\text {ref }}$ to calculate the value of the OPD at each time $T_{\mathrm{PV}, K}$.

(a) Scientific signal $\left(I_{s}\right)$

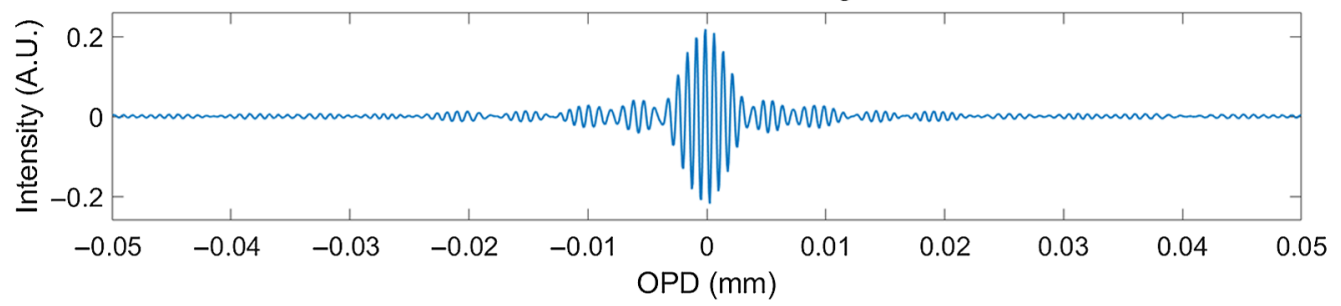

(b) Metrology signal $\left(I_{m}\right)$

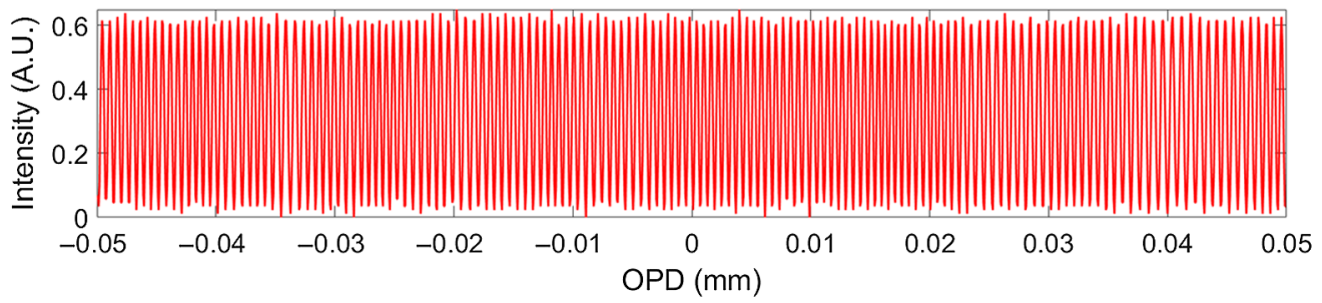

Fig. 5 (a) Scientific signal $I_{s}$ and (b) metrology signal simultaneously recorded during the measurement of the Sun spectrum.

(a)

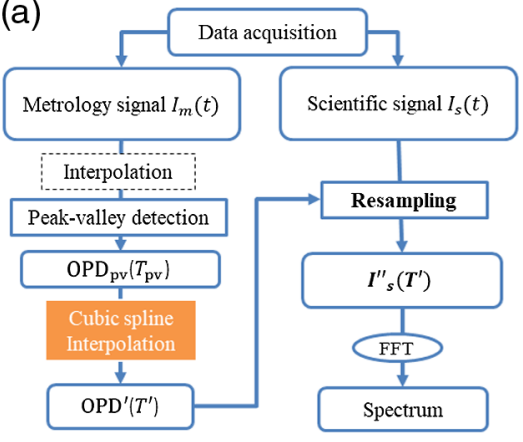

(b)

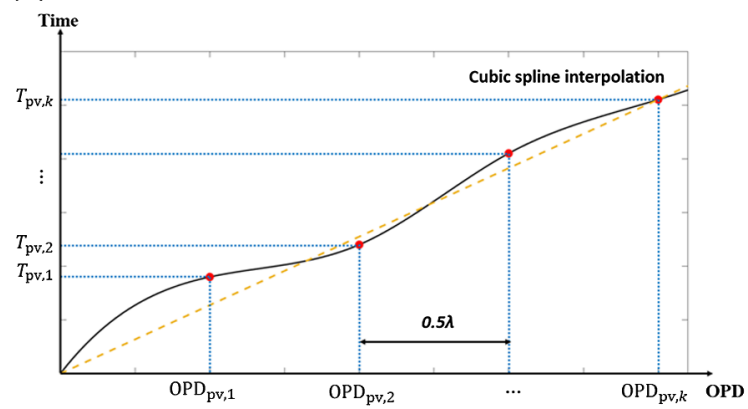

Fig. 6 (a) The flow chart of the data processing algorithm used to illustrate the resampling $l_{s}^{\prime}$ to be equally spaced intervals. (b) Time-position relation obtained from peak-valley detection is fitted with a cubic spline to evaluate the timestamp variation $T_{i}^{\prime}$. 
We use a cubic spline interpolation ${ }^{18}$ to calculate the value of the OPD at any time sample $T_{i}^{\prime}$ between $\left(T_{\mathrm{PV}, K-1}, \mathrm{OPD}_{\mathrm{PV}, K-1}\right)$ and $\left(T_{\mathrm{PV}, K}, \mathrm{OPD}_{\mathrm{PV}, K}\right)$ as described in Naylor et al. ${ }^{19}$ The output of the cubic spline interpolation is the time grid $T_{i}^{\prime}$ that corresponds to the uniform $\mathrm{OPD}_{i}^{\prime}$. We resample $I_{s}(t)$ using a cubic spline interpolation corresponding to the time grid $\operatorname{OPD}^{\prime}\left(T^{\prime}\right)$. The output of this interpolation is the signal $I_{s}^{\prime}$ that is sampled at an equidistance OPD value. Finally, the spectrum result is obtained after taking the fast Fourier transform to this interferogram resampling.

\section{Result}

\subsection{Contrast Variation Versus OPD}

The quality of the interference fringes is quantified by the fringe contrast defined $a s^{20}$ : $C=\left(I_{\max }-I_{\min }\right) /\left(I_{\max }+I_{\min }\right)$, where $I_{\max }$ and $I_{\min }$ are the maximum and minimum intensities of the fringe modulation, respectively. We measured the variation of the contrast at $\lambda_{\text {ref }}$ by injecting the beam provided by the metrology source in the scientific fiber and measuring the signal

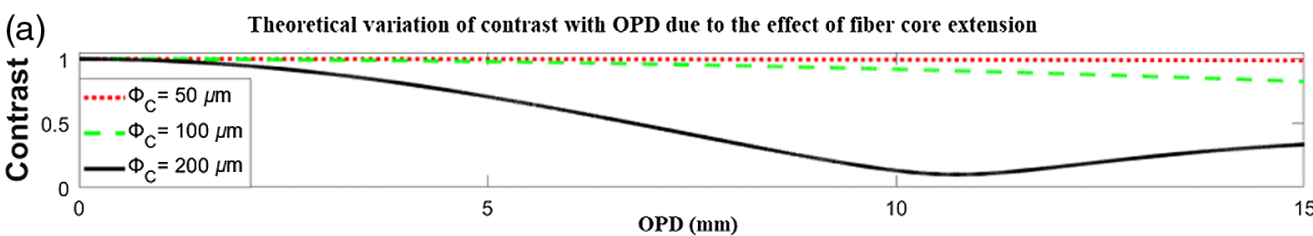

(b) Measured and theoretical variation of contrast with OPD

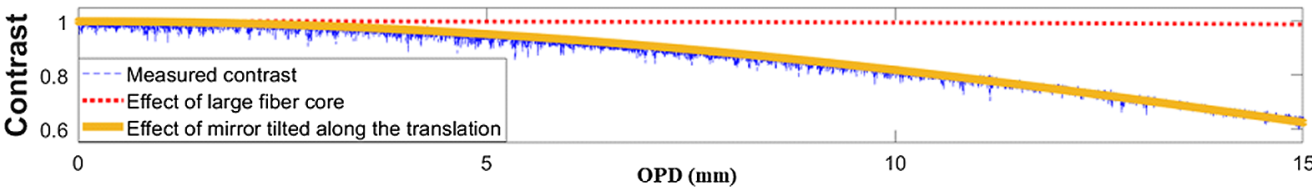

(c) Variation of image plane fringe pattern of fiber core 50 microns with captured at $I_{\max }(\mathrm{OPD})$ and $I_{\min }(\mathrm{OPD})$

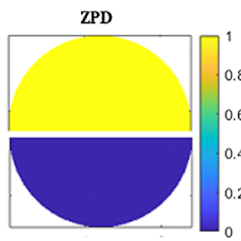

$\mathrm{ZPD}+2 / 2$

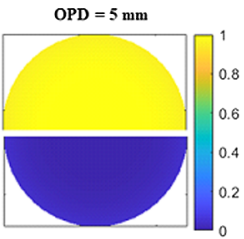

$\mathrm{OPD}=5 \mathrm{~mm}+\dot{j} / 2$

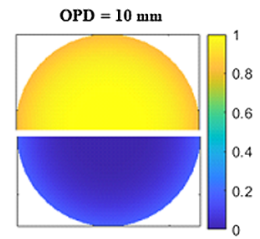

$\mathrm{OPD}=10 \mathrm{~mm}+\hat{\mu} / 2$

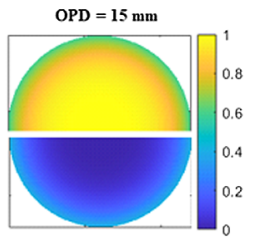

OPD $=15 \mathrm{~mm}+\% / 2$

(d) Variation of pupil plane fringe pattern due to dynamic mirror tip-tilt captured at $I_{\max }$ (OPD) and $I_{\min }$ (OPD)

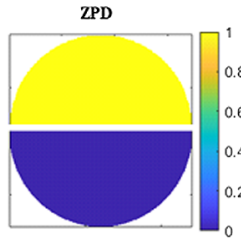

$\mathrm{ZPD}+j / 2$

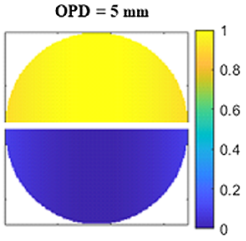

$\mathrm{OPD}=5 \mathrm{~mm}+\lambda / 2$

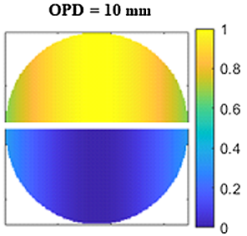

$\mathrm{OPD}=10 \mathrm{~mm}+\hat{k} / 2$

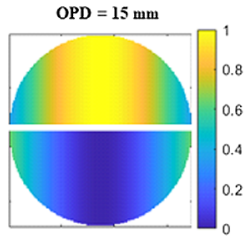

$\mathrm{OPD}=15 \mathrm{~mm}+\mathrm{z} / 2$

Fig. 7 (a) An illustration of contrast variation with respect to OPD for different fiber core diameters of 50,100 , and $200 \mu \mathrm{m}$ as plotted in the red dotted line, green dashed line, and black solid line, respectively. (b) The contrast variation with respect to OPD. Blue dashed line: experimental contrast measurement at $\lambda_{\text {ref }}$. Red dotted line: theoretical contrast variation due to the spatial extension of the fiber core. Yellow solid line: theoretical contrast variation due to a tilt of M1. (c) Distribution of maximum and minimum intensities of image planes induced by the spatial extension of the fiber core at ZPD and OPD $=5,10$, and $15 \mathrm{~mm}$. (d) Distribution of maximum and minimum intensities in the pupil planes induced by the $M 1$ tilt at $Z P D$ and $O P D=5,10$, and $15 \mathrm{~mm}$. 
provided by the detector while varying the OPD from the zero optical path difference (ZPD) to $\mathrm{OPD}_{\max }=1.5 \mathrm{~cm}$. The results are represented in blue solid line of Fig. 7(b).

We notice that the contrast varies from 1 to 0.6 between $\mathrm{ZPD}$ to $\mathrm{OPD}_{\max }$. We identified two possible origins of the contrast variation with the OPD: (1) The spatial extension of the fiber core and (2) the tilt of the dynamic mirror during the displacement. The impact of these on the contrast performance are investigated and discussed hereafter.

\subsection{Source Spatial Extension Impact on Contrast Performance}

The spatial extension of the fiber core introduces a variation of the OPD that impacts the contrast performance. ${ }^{21}$ We represent the fiber output face as a spatially incoherent source made of a disk uniformly illuminated of diameter $\Phi_{c}$. We set point M located in the plane of the fiber output face at the distance $y<\Phi_{c} / 2$ from the optical axis. The focal length of the collimator is $f_{\text {col }}=19 \mathrm{~mm}$. The angle between the beam transmitted by the collimator and the optical axis is $\theta \approx y / f_{\text {col }}$, as shown in Fig. 8(a).

The OPD of the beam emitted by $\mathrm{M}$ is ${ }^{21} \operatorname{OPD}_{\mathrm{M}}(L)=2 \cdot L \cos \left(y / f_{\text {col }}\right)$, where $L$ is the distance of the dynamic mirror that varies between $L_{\min }=0 \mathrm{~mm}$ at the ZPD and $L_{\max }=7.5 \mathrm{~mm}$ at $\mathrm{OPD}_{\max }$. The variation of OPD between the center and the edge of the fiber output face is $\delta \mathrm{OPD} \approx L \cdot\left(\Phi_{c} / 2 f_{\text {col }}\right)^{2}$. The maximum value of $\delta \mathrm{OPD}$ is reached at $L_{\max }$, where $\delta \mathrm{OPD}_{\max } \approx 30 \mathrm{~nm}$, which corresponds to $\lambda_{\text {ref }} / 20$. The intensity of the beam emitted by M and incident on the detector at wavelength $\lambda_{\text {ref }}=633.178 \mathrm{~nm}$ is

$$
\left.I_{\mathrm{M}}(y, D)=E_{0}^{2}\left[1+\cos \left(2 \pi \cdot \mathrm{OPD}_{\mathrm{M}}\right) / \lambda_{\text {ref }}\right)\right],
$$

where $E_{0}$ is the amplitude of the electromagnetic field. The source is spatially incoherent, and we deduce that the intensity detected at the mirror position $L$ is the integral of intensity emitted by each point of the fiber output face.

The results of contrast loss over the range between $\mathrm{ZPD}$ and $\mathrm{OPD}_{\max } 15 \mathrm{~mm}$ due to the effect of the fiber extension with varying the size of the fiber core are shown in Fig. 7(a). The contrast variations of fiber core diameter $\Phi_{c}=50,100$, and $200 \mu \mathrm{m}$ are plotted in the red dotted line, green dashed line, and black solid line, respectively. We found that the contrast decreases with respect to the spatially increasing fiber core diameter. Figure 7(c) shows the intensity distribution on the fiber output face for the fiber core diameter of $50 \mu \mathrm{m}$; note that the contrast is always close to 1 , which is used in the current system.

\subsection{Tip-Tilt of the Dynamic Mirror Impact on Contrast Performance}

The dynamic mirror M1 is mounted on a commercial translating stage with a pitch and roll that induce a tilt during M1 translation. We assume that the tilt $\alpha$ of M1 is around the vertical axis

(a)

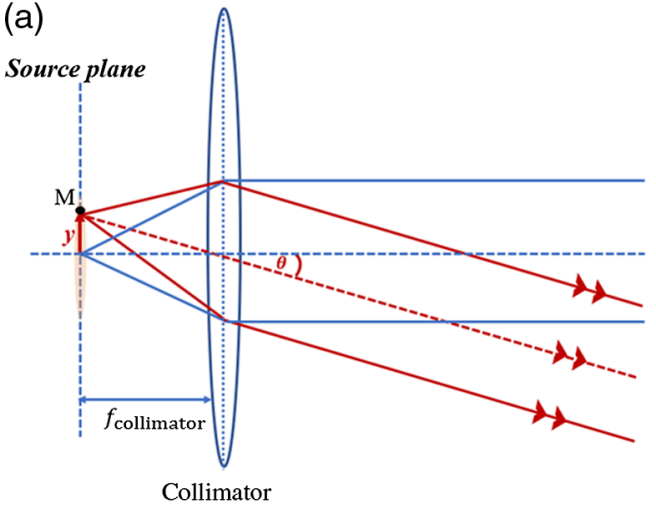

(b)

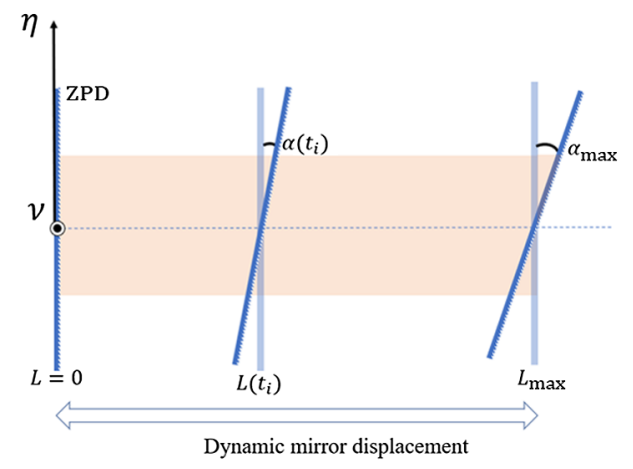

Fig. 8 (a) Illustration of ray propagating to the system with the angle of incident due to the effect of extension of fiber core. (b) Schematic of the linear tilt induced during the displacement of the dynamic mirror. 
$(O, \eta)$ and that $\alpha$ varies linearly with respect to the mirror position according to the following law: $\alpha=L \alpha_{\max }$, as shown in Fig. 8(b).

The intensity of the beam incident on the detector by point $\mathrm{M}$ located at coordinate $(\nu, \eta)$ at time $t_{i}$ is

$$
I_{\mathrm{M}}(\nu, D)=E_{0}^{2}\left[1+\cos \left(2 \pi \cdot \mathrm{OPD}_{\mathrm{M}}(\nu, D) / \lambda_{\mathrm{ref}}\right)\right]
$$

where

$$
\operatorname{OPD}_{\mathrm{M}}(\nu, D)=2 D\left(1+\alpha_{\max } \nu\right) .
$$

The intensity incident on the detector is calculated by the integral of the intensity distributed in the pupil plane. The yellow solid line in Fig. 7(b) represents the theoretical variation of the contrast due to the M1 tilt in the case of $\alpha_{\max }=21.9 \mu \mathrm{rad}$, corresponding to the illustrated intensity distributions over the pupil plane fringe pattern in Fig. 7(d).

As shown in Fig. 7(b), we notice that the theoretical contrast variation due to the M1 tilt fits perfectly to the measured contrast variation. This value is in very good agreement with the specification of the Newport M-ILS200CCL pitch, which is equal to $37 \mu \mathrm{rad}$ in the typical case.

\subsection{Instrument Line Shape}

Ideally, the measurement of the FTS ILS would consist of measuring the spectrum provided by the instrument for a monochromatic source. The only monochromatic source available for this measurement was the metrology laser. Unfortunately, our attempts to simultaneously inject the laser beam in the scientific and metrology fibers were not successful due to the interferences between the scientific and metrology beams.

We thus decided to make a preliminary measurement of the ILS of FTS system by (1) turning "ON" the metrology source, (2) removing the I-band filter located in front of the detector, and (3) measuring the fringes induced by the leakage of the metrology signal on the detector. This is done while displacing the dynamic mirror to adjust the OPD between $-\mathrm{OPD}_{\max }$ and $+\mathrm{OPD}_{\max }$.

It is important to mention that this ILS does not include the effect of the scientific fiber on the ILS. However, as mentioned in the previous section, the fiber core diameter has a negligible effect on the contrast, and the contrast is mostly driven by the tip-tilt of the dynamic mirror during the displacement. We know that metrology follows an optical path close to the scientific beam, and we thus decided to use it as a first approximation measurement to estimate the ILS of the prototype.

We calculated the theoretical ILS without the mirror tilt effect (ILS theo $)$ and with an effect of mirror tilt (ILS $\mathrm{S}_{\text {theo,tilt }}$ ). In the optical frequency domain, the frequency is noted as $\sigma=1 / \lambda$. The theoretical FWHM of $\mathrm{ILS}_{\text {theo }}$ is $^{22} \delta \sigma=1.207 / \mathrm{OPD}_{\max } \approx 0.80 \mathrm{~cm}^{-1}$ with $\delta \lambda \approx \lambda^{2} \delta \lambda$ in the wavelength domain. At the wavelength $\lambda=633.178 \mathrm{~nm}$, we deduce that $\delta \lambda_{\text {theo }} \approx 0.032 \mathrm{~nm}$ and thus the theoretical $R_{\text {theo }}=\lambda / \delta \lambda_{\text {theo }} \approx 19,744$. Figure 9 shows the theoretical shape of $\mathrm{ILS}_{\text {theo }}$ in a blue dotted line and the shape of $\operatorname{ILS}_{\text {theo,tilt }}$ in a yellow dashed line with a $\mathrm{FWHM}_{\text {theo,tilt }}=0.033 \mathrm{~nm}$. We notice that the central wavelength of the measured ILS, as plotted in the red solid line of Fig. 9, is thus different by $1.5 \mathrm{pm}$ from the theoretical central wavelength. The potential contributors to this difference are (1) laser stability of $2 \mathrm{pm}^{23}$ and (2) tuning error of the laser. ${ }^{22}$ The FWHM is measured as $\mathrm{FWHM}_{\mathrm{ILSmeas}}=0.033 \mathrm{~nm}$ (the same as $\left.\mathrm{FWHM}_{\text {theo,tilt }}\right)$, thus providing $R_{\text {meas }} \approx 19,609$.

\subsection{Solar Spectrum Measurement Setup, Result, and Analysis}

In this section, we present the results measured on January 27, 2021, from 3.30 PM to 4.30 PM local time in Thailand (GMT + 7). The sampling rate was set to $50 \mathrm{kHz}(50,000$ samples per second), and the scanning velocity of the dynamic mirror was $1.8 \mathrm{~mm} / \mathrm{s}$, which provided $\sim 10$ samples per fringes at the wavelength of $633.178 \mathrm{~nm}$. The amplitude of mirror displacement was $0.75 \mathrm{~cm}$ from the ZPD position, thus providing an $\mathrm{OPD}_{\max }$ of $1.5 \mathrm{~cm}$. The full scan between 
Artsang et al.: Design and laboratory performance of a fiber-fed Fourier transform spectrograph...

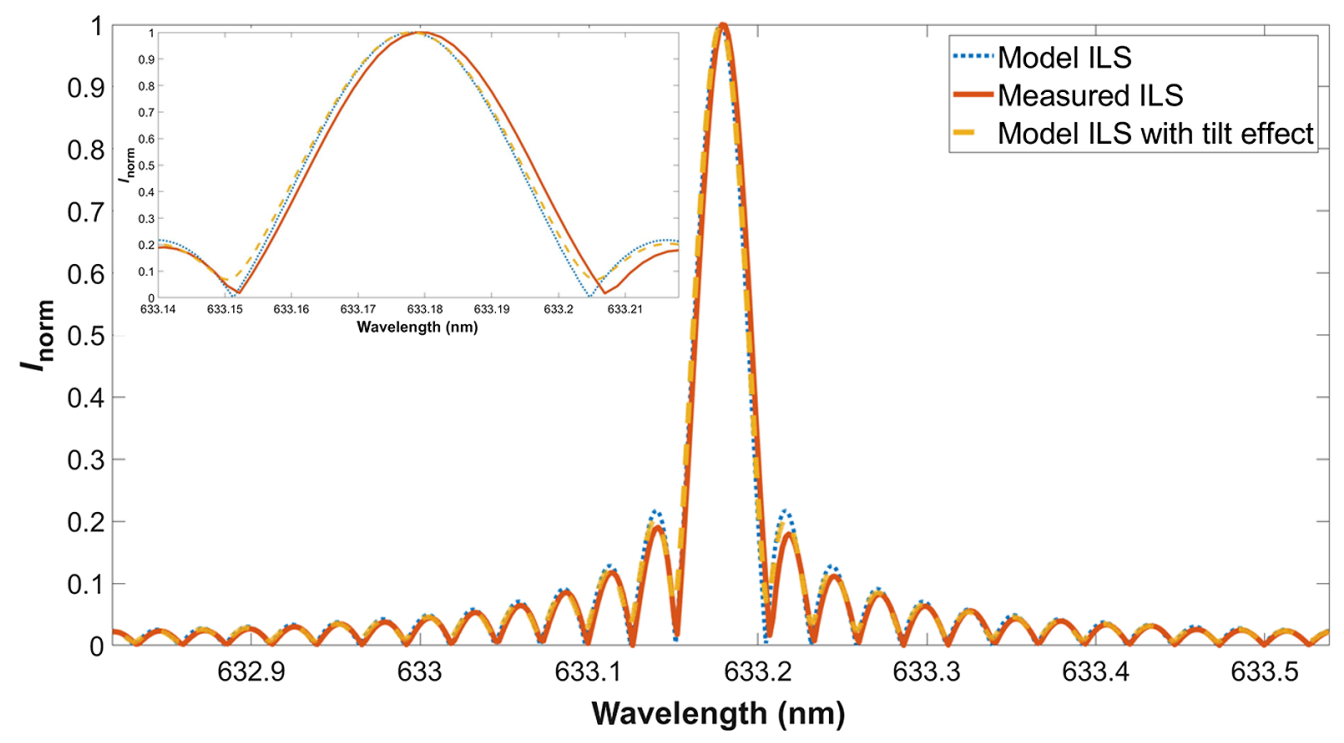

Fig. 9 The three plots of ILS with the zoom in (inset plot) at central wavelength $633.178 \mathrm{~nm}$. Blue dotted line: ILS theo (without tilt effect). Red solid line: The measured ILS from of metrology leakage in the FTS system. Yellow dashed line: ILS theo,tilt

$-\mathrm{OPD}_{\max }$ and $\mathrm{OPD}_{\max }$ took $<10 \mathrm{~s}$ in each measurement. We measured 40 individual interferograms, which were processed individually using the method presented in Sec. 3. The final interferogram is the average of the 40 measured and processed interferograms.

Figure 10(a) shows the theoretical spectrum of atmospheric transmission and the Sun, and the raw spectrum and the shifted spectrum of the Sun measured with our prototype are shown in Figs. 10(b) and 10(c), respectively. The theoretical spectrum includes the contribution of the theoretical Sun spectrum and of the atmospheric absorption lines, or telluric lines as described hereafter. The theoretical solar spectrum was obtained from the Solar atmospheric model adopted parameters and abundance. ${ }^{24}$ The synthetic spectrum of the Sun was
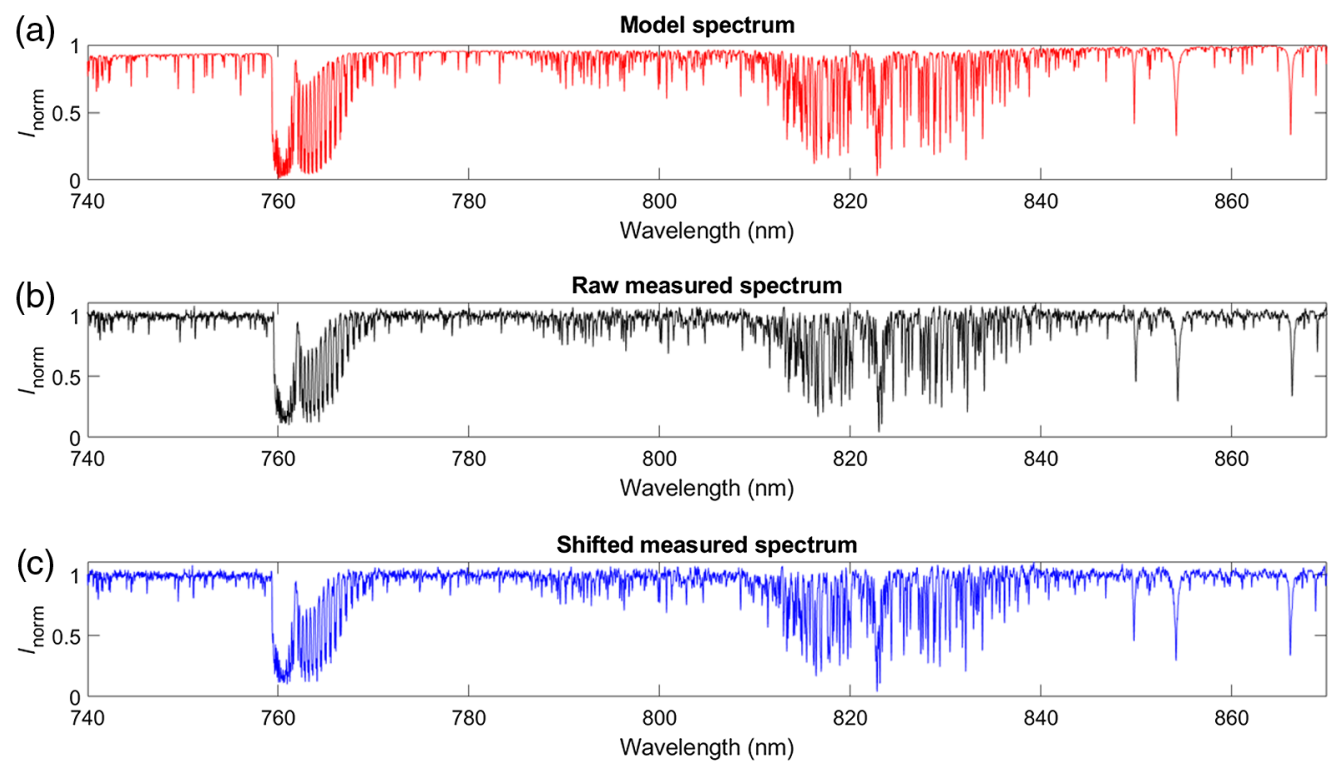

Fig. 10 The solar spectrum over the spectral range 740 to $870 \mathrm{~nm}$. (a) The model spectrum created from the convolution between the theoretical spectrum of the atmospheric transmission and the Sun. (b) The raw measured spectrum and (c) the measured spectrum with the instrumental shift. 
generated using SYNTHE code. ${ }^{25}$ The observed solar spectrum was shifted due to the Doppler shift from the Earth's barycentric motion, gravitational redshift, and instrumental shift.

The model of telluric lines was generated from a web-based service named Transmissions of the AtmosPhere for AStronomical (TAPAS) data. ${ }^{26}$ TAPAS generates atmospheric lines using LBLRTM code,${ }^{27}$ HITRAN molecular lines, ${ }^{28}$ and atmospheric profiles stored in the ETHER database, a French Atmospheric Chemistry Data Center ETHER (CNES and CNRS).

Although the input parameters were chosen to be matched with the observing condition, we expect to see some differences in the line depth and position between the measured spectrum and the theoretical model. The main cause of the differences comes from water molecules as they are highly variable and inhomogeneous in the atmosphere. The available atmospheric data in the database that TAPAS uses for generating a model are not available every moment, and it has to interpolate between data from the closet available times. In addition, the current version of TAPAS does not take into account the wavelength shift caused by winds. There are also some discrepancies in the width and shape of individual lines because the actual ILSF is different from the assumed Gaussian shape of the TAPAS. Nevertheless, in this study, the atmospheric transmission lines were used for instrumental calibration; the differences in the line depth, width, shape, and wavelength shift can be neglected for evaluating the current performance of our FTS spectrograph. ${ }^{29}$

The instrumental corrections were determined from the wavelength-shift of telluric lines between observed and theoretical transmission spectrum. Using the atmospheric transmission line in the $\mathrm{O}_{2}$ A-band (759 to $\left.772 \mathrm{~nm}\right)$ and $\mathrm{H}_{2} \mathrm{O}(810$ to $840 \mathrm{~nm}$ ) region as a reference, we derived the instrumental shift of the FTS in frequency $(1 / \lambda)$ of $3.3 \mathrm{~cm}^{-1}$, which is implemented on the measured spectrum and represented in the blue solid line of Fig. 10(c). The barycentric velocity of the Sun during the observation $\left(\mathrm{RA}=20^{\mathrm{h}} 30^{\mathrm{m}} 55^{\mathrm{s}} .69\right.$, DEC $=$ $-18^{\circ} 26^{\prime} 17^{\prime \prime} .2$ ) was calculated to be -482 to $-551 \mathrm{~m} / \mathrm{s}$ using the barycorrpy Python package, ${ }^{30}$ and the gravitational redshift of $638 \pm 6 \mathrm{~m} / \mathrm{s}$ determined by Hernández et al. ${ }^{31}$ was adopted.

Figure 11 shows the regions of absorption lines between the theoretical spectrum in the red dotted line and the raw measured spectrum (non-shifted) in the black dashed line. The two regions of (1) the $\mathrm{O}_{2}$ A-band (759 to $772 \mathrm{~nm}$ ) are shown in Fig. 11(a) and (2) the prominent
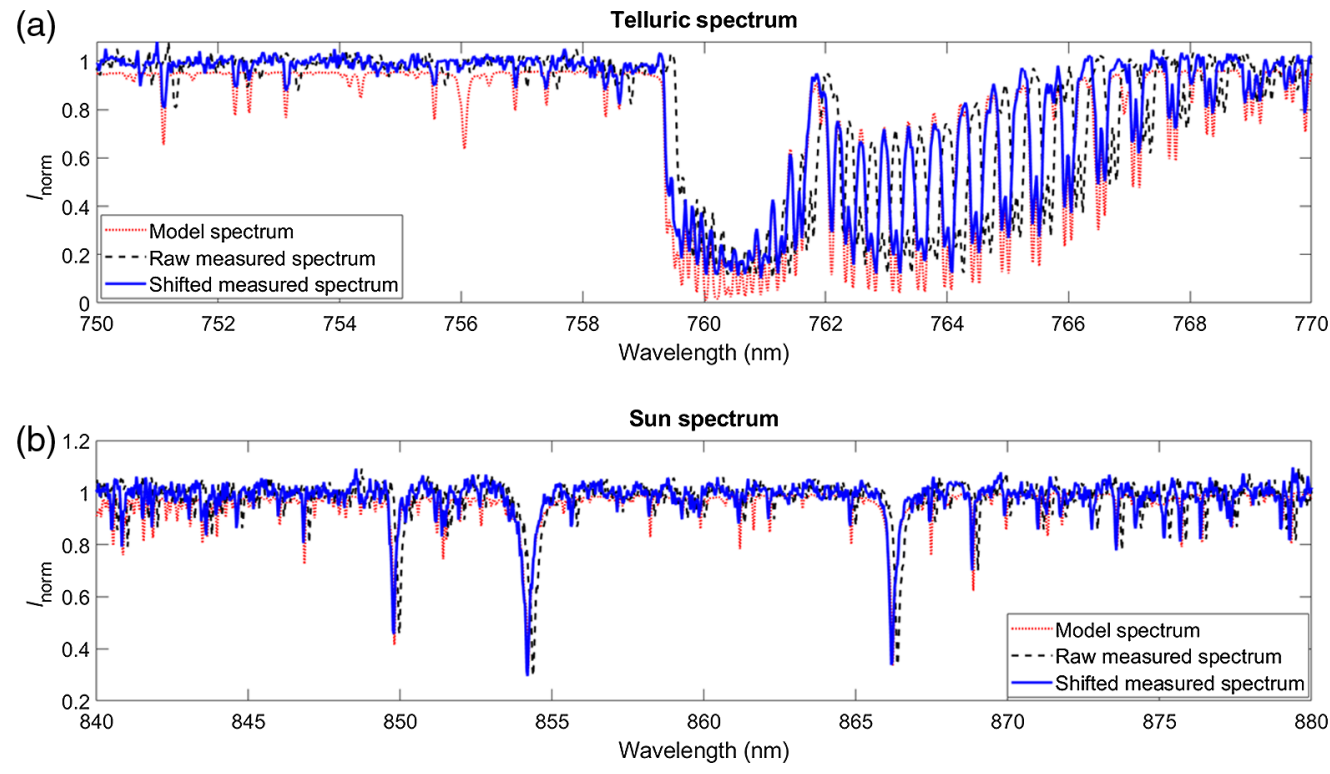

Fig. 11 The comparison of the model spectrum (red dotted line), the raw measured spectrum (black dashed line), and the shifted measured spectrum (blue solid line). (a) The region of telluric absorption lines over 750 to $770 \mathrm{~nm}$. (b) The region of Sun absorption lines over 840 to $880 \mathrm{~nm}$. 
Ca-II triplet absorption lines $(849.8,854.2$, and $866.2 \mathrm{~nm})$ are shown in Fig. 11(b). The measured spectrum after applying the correction of the instrumental and Doppler spectral shifts are presented in the blue solid line.

\subsection{Spectral Resolution and Radiometric Quality}

Figure 12 shows the results of four absorption lines including envelope regions, which are considered to be isolated and not blended, to not have a too strong and too faint absorption line, and to not overlap with the telluric lines for the spectral resolution and SNR determination. First, as shown in Fig. 12(a), we present the region of the small intrinsic absorption line of iron (Fe-I) at wavelength $846.8 \mathrm{~nm}$, which is represented as the detection limit of the instrument and determines the wavelength resolved in spectral resolution. Second, we show the three distinctive Ca-II triplet absorption lines at wavelength $849.8 \mathrm{~nm}$ in Fig. 12(b), $854.2 \mathrm{~nm}$ in Fig. 12(c), and $866.2 \mathrm{~nm}$ in Fig. 12(d). These three strong absorption lines are mostly used to study and investigate the magnetic field and temperature structure in Sun observations. ${ }^{32}$ Table 1 summarizes the central wavelength, FWHM, and SNR measured on Fe-I and Ca-II triplet absorption lines. The SNR is defined as the ratio between the depth of absorption line $\left(I_{\mathrm{abs}}\right)$, and the standard deviation of its noise envelop $(\sigma) \mathrm{SNR}=I_{\mathrm{abs}} / \sigma$, as shown in Fig. 13.

The results presented in Fig. 12 and in Table 1 show that our spectrograph is clearly able to detect spectral lines with widths varying between $0.0562 \mathrm{~nm}$ (Fe-I) and $0.185 \mathrm{~nm}$ (second Ca-II). The overall profile, depth, and FWHM of the Ca-II absorption lines were well measured. We thus deduce that our prototype is able to correctly measure some spectral lines with a width typically

(a)

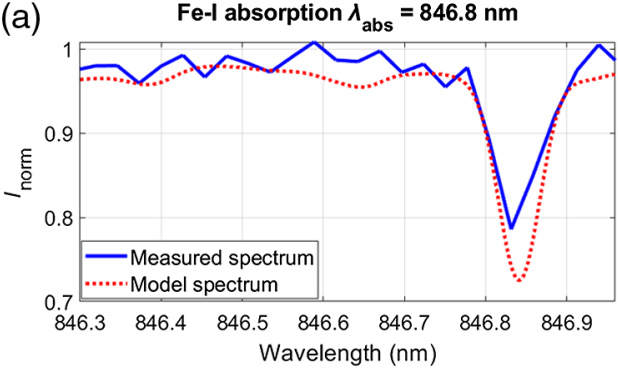

(c)

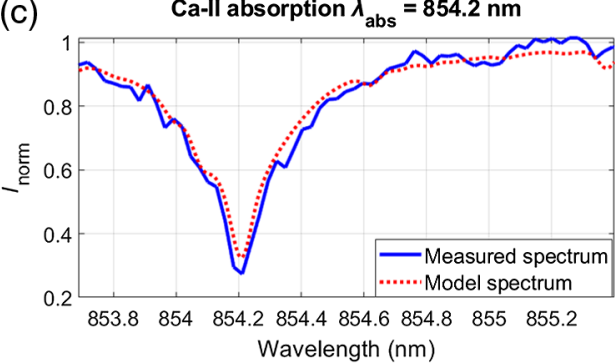

(b)

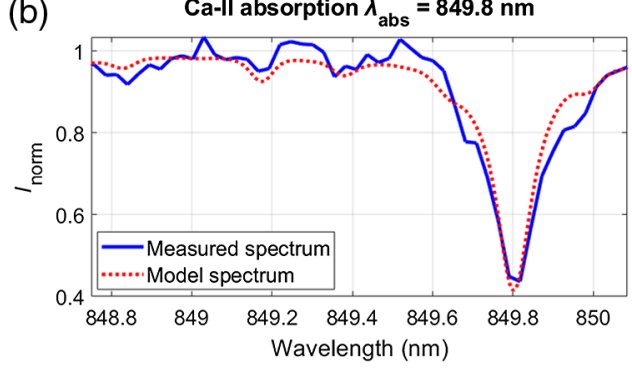

(d)

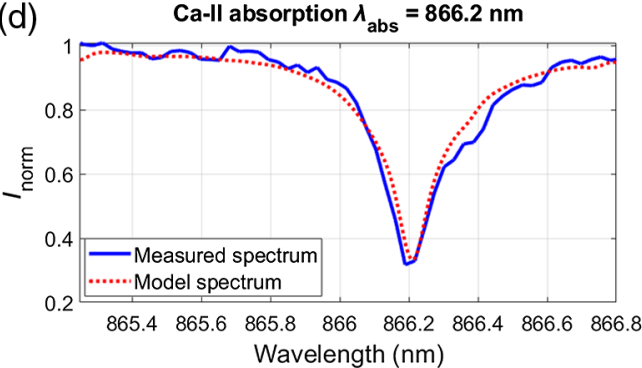

Fig. 12 (a) Fe-I absorption line at $846.8 \mathrm{~nm}$. (b) First Ca-Il absorption at central wavelength $849.8 \mathrm{~nm}$. (c) Second Ca-II absorption at central wavelength $854.2 \mathrm{~nm}$. (d) Third Ca-II absorption at central wavelength $866.2 \mathrm{~nm}$.

Table 1 The spectral qualities of each absorption line in Fig. 12.

\begin{tabular}{lcccc}
\hline \hline & (a) Fe-I & (b) First Ca-II & (c) Second Ca-II & (d) Third Ca-II \\
\hline Central wavelength & $846.8 \mathrm{~nm}$ & $849.8 \mathrm{~nm}$ & $854.2 \mathrm{~nm}$ & $866.2 \mathrm{~nm}$ \\
FWHM & $0.0562 \mathrm{~nm}$ & $0.125 \mathrm{~nm}$ & $0.185 \mathrm{~nm}$ & $0.181 \mathrm{~nm}$ \\
SNR & 17.6 & 24.9 & 22.9 & 34.9 \\
\hline \hline
\end{tabular}




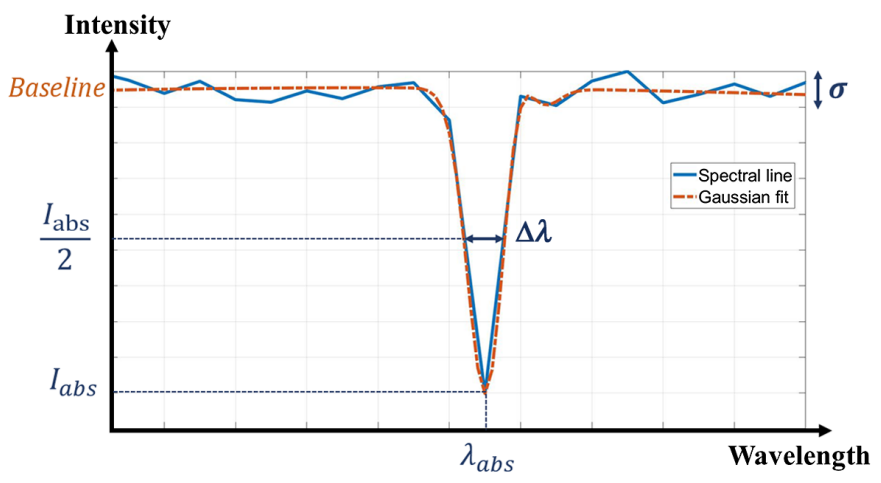

Fig. 13 Illustration of the region of the absorption line for calculating spectral resolution and SNR.

larger than $0.125 \mathrm{~nm}$. The limit of detection of our instrument seems to be reached on the Fe-I absorption line of FWHM equal to $0.056 \mathrm{~nm}$ corresponding to $R \approx 15,000$. We were able to detect the absorption line with an SNR close to 20, but we failed in measuring the correct depth and reproducing the shape of the Fe-I absorption line. We conclude from these observations that (1) the spectral resolution of our FTS is close to 15,000 over the spectral domain [750 nm, $900 \mathrm{~nm}$ ] and (2) the SNR is close to 20 at the limit of resolution for an incident flux of about $1 \mu \mathrm{W}$.

\section{Conclusion}

This paper presents a fiber-fed FTS that is built from off-the-shelf optical components. The instrument concept relies on a dual-output Michelson interferometer that provides a high throughput and is insensitive to fiber modal noise. The cost and size of the FTS prototype are comparable with that of a commercially available FTS-based spectrum analyzer, for example, the OSA201C from Thorlabs. However, the custom developed FTS was specifically design for astronomical observation. The size of the proposed system is about half size of the middleresolution fiber-fed Echelle spectrograph (MRES) of the TNT. The cost of the developed FTS system would be about 10 times lower as compared with that of conventional spectrograph such as MRES that currently are used by the TNT. The total throughput obtained from the developed FTS is $42 \%$ at wavelength $800 \mathrm{~nm}$, which is higher than the throughput of MRES that is approximately $26 \%$ at the same wavelength.

We investigated the contrast performance of the FTS system with two assumptions of the spatial extension of the fiber and the tilt of the dynamic mirror during the displacement and found that the contrast drop as a function of OPD is negligible for the developed FTS system. Based on the result in Fig. 7(b), the contrast drop is currently dominated by the tilt of M1 induced by the translating stage.

Furthermore, we found that the spectral resolution obtained from the FWHM of the measured ILS is approximately 19,609 at a wavelength of $633.178 \mathrm{~nm}$. The central wavelength of the measured ILS jittered in the range of $1.5 \mathrm{pm}$ from the theoretical central wavelength, which may be caused by laser stability and a tuning error.

Finally, the performance of the developed system was verified by observing the Sun's spectrum. The measured spectrum of the Sun in the range of 740 to $880 \mathrm{~nm}$ was compared with the model atmospheric transmission and synthetic spectrum of the Sun with identified telluric and atomic absorption lines. We found that this prototype is able to observe the solar spectrum with a spectral resolution and SNR close to 15,000 and 20, respectively.

The next development steps will aim at reaching $R \approx 70,000$ and an SNR higher than 10 on stars of magnitude six over the full spectral range [400 nm, $1 \mu \mathrm{m}]$. The upgrades to reach these objectives will consist of implementing a phase-lock amplifier, increasing the integration time to $1 \mathrm{~ms}$, and installing a high-performance air-bearing translating stage. This upgrade will provide the stability and the quality of movement of the dynamic mirror required to measure good quality fringes and to increase the contrast of the interferometer with the objective to reach $C>0.3$ at a maximum OPD of $50 \mathrm{~mm}$. 
Artsang et al.: Design and laboratory performance of a fiber-fed Fourier transform spectrograph...

\section{Appendix A: Components Description}

The part lists of FTS components and their manufacturer catalog numbers are presented in Table 2 for the scientific channel and Table 3 for the metrology channel.

\subsection{Scientific Channel}

Table 2 List of the components of the scientific channel in the FTS system.

\begin{tabular}{|c|c|c|}
\hline Component & Specification & Manufacturer catalog number \\
\hline Scientific fiber & Multimode fiber, $0.22 \mathrm{NA}, \varnothing 50 \mu \mathrm{m}$ core & Thorlabs FG050LGA \\
\hline Collimator L1 & $\begin{array}{l}\text { Achromatic doublets, AR-coated } \\
f=19.0 \mathrm{~mm}, \varnothing 1 / 2^{\prime \prime}\end{array}$ & Thorlabs AC127-019-B \\
\hline BS & 50:50 Nonpolarizing cube, AR-coated & Thorlabs BS014 \\
\hline Translating stage & $\begin{array}{l}\text { Motion controller with USB interface } \\
\text { - Motorized linear stage, } 200 \mathrm{~mm} \text {, DC motor }\end{array}$ & $\begin{array}{l}\text { Newport ESP } 301 \\
- \text { Newport M-ILS200CCL }\end{array}$ \\
\hline Mirror M1, M2 & Protected gold mirror & Thorlabs PF10-03-M01 \\
\hline Spectral filter & I-band region (700 to $900 \mathrm{~nm}$ ) & Astrodon Photometrics \\
\hline Prism P1 & $\begin{array}{l}\text { Right-angle prism, AR coating on } \\
\text { hypotenuse }\end{array}$ & Thorlabs PS908H-B \\
\hline Detector & $\begin{array}{l}\text { Balanced receiver with a large } 8 \mathrm{~mm} \\
\text { diameter }\end{array}$ & Newport 2307 \\
\hline
\end{tabular}

\subsection{Metrology Channel}

Table 3 List of the components of the metrology channel in the FTS system.

\begin{tabular}{lll}
\hline \hline Component & \multicolumn{1}{c}{ Specification } & Manufacturer catalog number \\
\hline Metrology source & Single longitudinal mode (SLM_52371_2-258) & Edmund optics \\
Metrology fiber & $\begin{array}{l}\text { Single-mode fiber yellow reinforced } \varnothing 3 \mathrm{~mm} \\
\text { Furcation tubing }\end{array}$ & Thorlabs FT030-Y \\
Collimator L2 & Achromatic doublets, AR-coated $f=50.0 \mathrm{~mm}, \varnothing 1^{\prime \prime}$ & Thorlabs AC254-050-A-ML \\
Prism P2 & Right-angle prism, AR coating on hypotenuse & Thorlabs PS908H-B \\
Prism P3 & Right-angle prism, AR coating on hypotenuse & Thorlabs PS908H-B \\
Detector & $\begin{array}{l}\text { Optical receiver with a large } 5.8 \text { mm diameter } \\
\text { Silicon detector }\end{array}$ & Newport 2032 \\
\hline \hline
\end{tabular}

\section{Acknowledgments}

This project was co-funded by the National Astronomical Research Institute of Thailand (NARIT), Suranaree University of Technology (SUT), and Thailand Science Research and Innovation (TSRI). The authors would like to thank Dr. Anthony Berdeu and Dr. Supachai Awiphan from National Astronomical Research Institute of Thailand (NARIT) and Dr. Sorawis Sangtawesin from Suranaree University of Technology (SUT) for the constructive discussions. Pornapa Artsang would like to acknowledge the Development and Promotion of Science and Technology Talents Project (DPST) scholarship for her PhD study. 
Artsang et al.: Design and laboratory performance of a fiber-fed Fourier transform spectrograph...

\section{References}

1. P. Massey and M. M. Hanson, Astronomical Spectroscopy, p. 35, Springer, Dordrecht (2013).

2. J. P. Maillard et al., "Integral wide-field spectroscopy in astronomy: the imaging FTS solution," Exp. Astron. 35, 527-559 (2013).

3. L. Drissen et al., "Imaging Fourier transform spectroscopy for astronomy," in Fourier Transforms-New Analytical Approaches and FTIR Strategies, G. S. Nikolić et al., Ed., pp. 493-520, InTech, Rijeka, Croatia (2011).

4. C. A. Pilachowski et al., "An archive of spectra from the Mayall Fourier transform spectrometer at Kitt Peak," Publ. Astron. Soc. Pac. 129, 1-11 (2017).

5. L. Drissen et al., "SITELLE: A Primer," https://www.cfht.hawaii.edu/Instruments/Sitelle/ FTS-primer-Jan16.pdf (2021).

6. F. Grandmont, L. Drissen, and M. Baril, "The SITELLE optical imaging Fourier transform spectrometer at the Canada-France-Hawaii telescope," in Light, Energy and Environ., FW5C.1, OSA, Leipzig (2016).

7. C. Pilachowski et al., "High-resolution spectrographs for large telescopes," Publ. Astron. Soc. Pac. 107, 983-989 (1995).

8. A. Chakraborty et al., "The PRL stabilized high-resolution Echelle fiber-fed spectrograph: instrument description and first radial velocity results," Publ. Astron. Soc. Pac. 126, 133-147 (2014).

9. F. Pepe et al., "HARPS: a new high-resolution spectrograph for the search of extrasolar planets," Proc. SPIE 4008, 582-592 (2000).

10. F. Rodler and G. Lo Curto, "HARPS User Manual," European Southern Observatory (2019).

11. F. Pike et al., "Modal noise mitigation for high-precision spectroscopy using a photonic reformatter," Mon. Not. R. Astron. Soc. 497, 3713-3725 (2020).

12. C. Buisset et al., "Optical and mechanical design and characterization of the new baffle for the 2.4-m Thai National Telescope," Proc. SPIE 9626, 96262F (2015).

13. Thorlabs Inc., "Step index multimode fiber: 0.22 NA," https://www.thorlabs.com/navigation .cfm?guide_id=2331 (Retrieved 20 May 2018).

14. C. Buisset et al., "Progress on the prevention of stray light and diffraction effects on the Thai National Telescope," Proc. SPIE 9626, 96262E (2015).

15. E. Lhospice et al., "EXOhSPEC folded design optimization and performance estimation," Proc. SPIE 11117, 111170Z (2019).

16. S. A. Roy, J. Genest, and P. Giaccari, "Hybrid sampling approach for imaging Fourier-transform spectrometry," Appl. Opt. 46, 8482-8487 (2007).

17. R. Aliaga, "Real-time estimation of zero crossings of sampled signals for timing using cubic spline interpolation," IEEE Trans. Nucl. Sci. 64, 2414-2422 (2017).

18. J. W. Brault, "New approach to high-precision Fourier transform spectrometer design," Appl. Opt. 35, 2891-2896 (1996).

19. D. Naylor et al., "Data processing pipeline for a time-sampled imaging Fourier transform spectrometer," Proc. SPIE 5546, 61-72 (2004).

20. T. D. Milster and N. A. Beaudry, "Coherence and Fringe Localization," https://www.tau.ac .il/ lab3/OPTICFIBERS/Coherence\%20and\%20Fringe.pdf (2021).

21. P. R. Griffiths, J. A. De Haseth, and J. D. Winefordner, Fourier Transform Infrared Spectrometry, 2nd ed., Wiley, New Jersey (2007).

22. A. Lacan et al., "A static Fourier transform spectrometer for atmospheric sounding: concept and experimental implementation," Opt. Express 18, 8311-8331 (2010).

23. P. Inc., "Single longitudinal mode SLM BOXX operating manual," http://www.pd-ld.com.

24. M. Asplund, N. Grevesse, and A. J. Sauval, "The solar chemical composition," in ASP Conf. Ser., Vol. 336, p. 25 (2005).

25. R. Kurucz, "SYNTHE spectrum synthesis programs and line data," CD-ROM No. 1-18 (1993).

26. J. L. Bertaux et al., "TAPAS, a web-based service of atmospheric transmission computation for astronomy," Astron. Astrophys. 564, A46 (2014). 
27. S. A. Clough and M. J. Iacono, "Line-by-line calculation of atmospheric fluxes and cooling rates: 2. Application to carbon dioxide, ozone, methane, nitrous oxide and the halocarbons," J. Geophys. Res. 100, 16519-16535 (1995).

28. L. S. Rothman et al., "The HITRAN2012 molecular spectroscopic database," J. Quant. Spectrosc. Radiat. Transf. 130, 4-50 (2013).

29. D. V. Cotton, J. Bailey, and L. Kedziora-Chudczer, "Atmospheric modelling for the removal of telluric features from infrared planetary spectra," Mon. Not. R. Astron. Soc. 439, 387-399 (2014).

30. J. T. Wright and S. Kanodia, "Barycentric corrections for precise radial velocity measurements of sunlight," Planet. Sci. 1, 38 (2020).

31. J. I. G. Hernández et al., "The solar gravitational redshift from HARPS-LFC Moon spectra," Astron. Astrophys. 643, A146 (2020).

32. J. Rodriguez et al., "Physical properties of a sunspot chromosphere with umbral flashes," Astron. Astrophys. 556, A115 (2013).

Pornapa Artsang is a PhD candidate at Suranaree University of Technology and a research assistant at the National Astronomical Research Institute of Thailand. She received her BS degree in physics from Suranaree University of Technology with first-class honors in 2015. She was awarded a scholarship of the Development and Promotion of Science and Technology Talents Project (DPST) from Royal Government of Thailand. Her current research interests focus on optical systems for astronomical applications. She is a member of SPIE.

Panomsak Meemon is an associate professor at the School of Physics, Suranaree University of Technology (Thailand). His research involves the development of optical imaging systems, focusing on optical coherence tomography (OCT). He received his $\mathrm{PhD}$ in optics from the College of Optics and Photonics, University of Central Florida (United States). He is a member of SPIE.

Pakakaew Rittipruk is a postdoc researcher at the National Astronomical Research Institute of Thailand (NARIT). She received her PhD in astronomy and space science from Sejong University in 2019. Her current research interests are stellar astrophysics, spectroscopic observation, and spectral instrumentation.

Sirinrat Sithajan is a researcher at the National Astronomical Research Institute of Thailand (NARIT). She received her BS degree in physics from Mahidol University in 2007, her MS degrees in astronomy from the University of Massachusetts Amherst in 2011, and her PhD in astronomy from the University of Florida in 2018. She is the author of nine journal papers. Her current research interests include exoplanet detection and characterization. She is a member of SPIE.

Biographies of the other authors are not available. 